\title{
A PROCEDURAL SOLUTION TO MODEL ROMAN MASONRY STRUCTURES
}

\author{
V. Cappellini ${ }^{\text {a,c }}$, R. Saleri ${ }^{\text {b }}$, C. Stefani ${ }^{a}$, N. Nony ${ }^{a}$, L. De Luca ${ }^{a}$ \\ ${ }^{\mathrm{a}}$ UMR (CNRS/MCC) 3495 MAP-Gamsau - ENSA Marseille, France - (valeria.cappellini, chiara.stefani, nicolas.nony, \\ livio.deluca)@map.archi.fr \\ ${ }^{\mathrm{b}}$ UMR (CNRS/MCC) 3495 MAP-Aria - ENSA Lyon, France - renato.saleri@lyon.archi.fr \\ ${ }^{c}$ DARTE - University of Reggio Calabria, Reggio Calabria, Italy
}

KEY WORDS: Procedural Modelling, Archaeological Data Representation, Photogrammetric Techniques, Parametric Elements

\begin{abstract}
:
The paper will describe a new approach based on the development of a procedural modelling methodology for archaeological data representation. This is a custom-designed solution based on the recognition of the rules belonging to the construction methods used in roman times. We have conceived a tool for 3D reconstruction of masonry structures starting from photogrammetric surveying. Our protocol considers different steps. Firstly we have focused on the classification of opus based on the basic interconnections that can lead to a descriptive system used for their unequivocal identification and design. Secondly, we have chosen an automatic, accurate, flexible and open-source photogrammetric pipeline named Pastis Apero Micmac - PAM, developed by IGN (Paris). We have employed it to generate ortho-images from non-oriented images, using a user-friendly interface implemented by CNRS Marseille (France). Thirdly, the masonry elements are created in parametric and interactive way, and finally they are adapted to the photogrammetric data.

The presented application, currently under construction, is developed with an open source programming language called Processing, useful for visual, animated or static, 2D or 3D, interactive creations. Using this computer language, a Java environment has been developed. Therefore, even if the procedural modelling reveals an accuracy level inferior to the one obtained by manual modelling (brick by brick), this method can be useful when taking into account the static evaluation on buildings (requiring quantitative aspects) and metric measures for restoration purposes.
\end{abstract}

\section{INTRODUCTION}

\subsection{Procedural Modelling for Virtual Reconstruction of Archaeological Sites.}

3D modelling, based on range sensors and imaging devices, is currently used for many different purposes such as historical documentation, digital preservation and conservation, virtual reality/computer graphics applications, and so on. Nowadays many research groups have created complete, high-quality, digital models of Cultural Heritage objects and sites (PierrotDeseilligny et al., 2011). Moreover, recent studies demonstrate that the best approach to achieve a detailed digital model is the combination of different 3D modeling techniques (Guidi et al., 2008). Particularly in the contest of archaeological survey, the pursuit of precision and accuracy is the first goal engaged by researchers and surveyors. But despite all these potential applications, 3D modelling of an historic monument is a tedious and time-consuming process (Chevrier et al., 2011) and it presents the limit to represent only visible or existing parts of an object (Ferdani et al., 2011).

For this reason, in the last 20 years a different methodological approach has been applied for the creation of Cultural Heritage objects, giving life to a modern field of Archaeology called Virtual Archaeology (Pescarin, 2009). This discipline faces the philological problem of the virtual reconstruction of ancient potential contexts and landscapes of archaeological sites. Its approach is based on literary references and it is validate by specialists. In other words, the principal aim of Virtual
Archaelogy is to interpret, to understand, and to communicate the different data relative to the archaeological sites.

This process starts from: 1) the data acquisition from different sources such as laser scanning, image based modelling, literary reference et so on; 2) goes through to 3D models creations by 3D and procedural modelling techniques; 3 ) and arrives at performing computer simulations and interactive visualisations to gather different information levels concerning the archaeological sites.

The communications mediums are different: real-time $3 \mathrm{~d}$ and multimedia applications, augmented reality, 3D stereoscopic short films and so on.

In particular the procedural modelling, employed for the reconstruction of ancient urban environments of archaeological sites, is a method for creating the objects geometry according to automatic or semi-automatic criteria. It is recommended to model a high number of entities that are recurring in space at a random, auto-similar and repetitive way.

In the first time, the procedural modelling was been used to produce textures and complex organic geometries. Only recently this procedure is employed to create animation, computer simulation of natural phenomena and finally architectonical models and geometries.

Using a grammatical approach, the generation of different typologies is made possible by a scripting language.

In general this procedure is divided in two steps: a) analytical step, identifying shapes and rules vocabulary, useful for planning operations; b) generative step, reproducing the architectonic and urban structures. 


\subsection{Related Works}

The potential applications of procedural modelling range from the singular architectonic elements to the urban plannings and virtual environments. Different approaches and solutions have been developed to model architectural objects, car components, furniture, traffic networks, buildings and so on.

In particular an interesting approach is represented by Generative Modeling Language (GML), a low level interpretive language for the description of the procedural shape. This language has been developed by the German computer scientist Sven Havemann within his $\mathrm{PhD}$ thesis (Havemann, 2005). He divides the existing 3D modeling methods in two classes: 1) the first class uses the 'list of primitives' approach, describing 3D objects and scenes as a combination of elementary geometric objects (points, triangles, Nurbs patches and so on); 2) and the second class is the group of procedural shape representations, including different methods such as shape grammar and L-system, shape programming language and several others. Havemann affirms none of these 3D modeling methods is entirely satisfactory. For this reason he proposes a new approach to represent the 3D models in procedural way using operations instead of objects. In fact the objects are created by several involved operations. The idea of generative modeling is to understand the shape of an object and produce it with not a list of elementary geometry elements but with a list of operations. Even if it represents an original approach to model the objects in parametric way, the lack of a user-friendly interface restricts to experts the use of this language, placing it out of everyone's reach.

In Archaeology, the introduction of procedural modelling urges the researchers to test new methodologies.

In particular, Muller proposes a system called Cityengine using a procedural approach to model cities (Parish et al., 2001; Muller et al., 2006; Haegler et al., 2009). Conceived for computer graphics applications, Cityengine is a system able to model a city starting from 2D information (GIS, plans and so on). Therefore procedural modelling of a city is based on union of different procedures (Ferdani et al., 2001): 1) image based, basing on terrain maps and aerial images of the buildings allocation; 2) L System, computer language representing the complexity of an organism or a building by few parameters and an growth algorithm; 3) Parametrical rules, giving the possibility to choose the detail and realism level.

Moreover, different steps are necessary to create a $3 \mathrm{D}$ city. 2D information is transformed in $3 \mathrm{D}$ streets, blocks and parcels. In these parcels are allocated the buildings created by $3 \mathrm{D}$ extrusion operations and the facades are textured, using a procedural approach based on L-system. It is possible to establish the shape grammar rules by scripts for the definition and the control of geometrical parameters and hierarchical relationships between the architectonic elements of buildings and their automatic generation. During the scripting step, a correlation matrix is created for giving a credible aspect to the model. This matrix assigns dimensions and different textures to the buildings in random way established by the user.

An interesting test case modelling by the CityEngine software is Procedural Pompeii. This project has been carried out within the European Project Cyberwalk.

The ancient city of Pompeii has been reconstructed mixing real surviving and excavation data with GIS information such as population density, land usage, street network and building footprints. The building geometries are created by shape grammar rules derived from photos and plans of remaining buildings, archaeological excavation data, and historical paintings. The Pompeii model has been completely generated with CityEngine.

The same methodology is adopted by VHLab CNR-ITABC to rebuild the ancient landscape of Bologna in Roman and Etruscan times (Ferdani et al., 2011). The approach of this project starts from the ruins $3 \mathrm{~d}$ acquisition by RBM and IBM techniques and then it resorts to procedural modelling to rebuild the potential aspect of ancient city in different ages.

These two test cases demonstrate that procedural modelling is especially used in archaeology to reconstruct ancient urban environments for communication, learning and educational purposes.

We have tested the ability of Cityengine software to create different opus typologies. The outcome is not really satisfying because we want to create the masonry in parametric and interactive way, and then adapting them to the photogrammetric data.

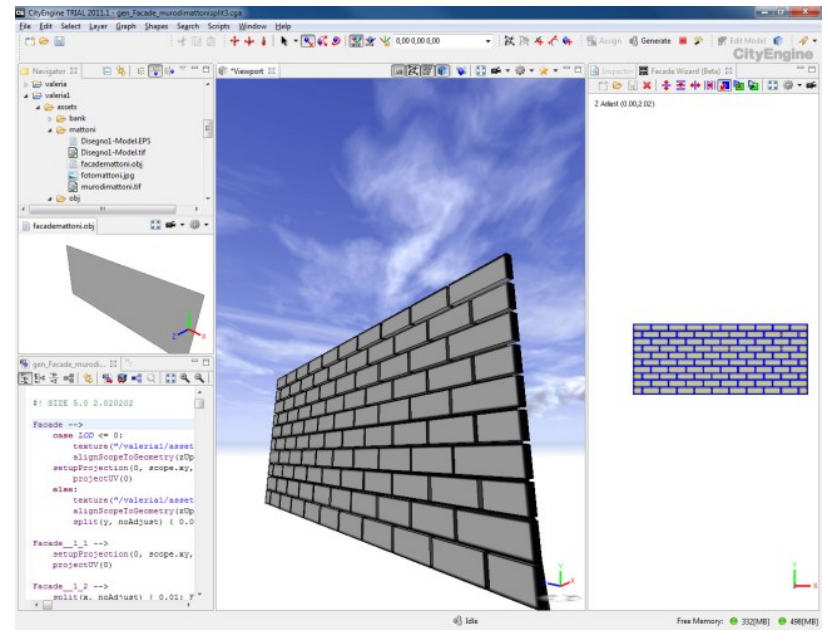

Figure 1. Cityengine test reproducing opus vittatum typology

\section{OUR APPROACH}

The proposed protocol is based on the development of a procedural modelling methodology for archaeological data representation.

The approach has been developed along three steps: 1) the semantic recognition of the rules belonging to the construction methods used in roman times; 2) the individuation of opus typologies on the masonry structures; 3 ) the creation of a parametric model describing the relation among geometric, semantic and quantitative data.

\subsection{Semantic Recognition of Roman Rules}

In procedural modelling, the geometry generation is automatic or semi-automatic. It is recommended to model a high number of entities that are recurring in space at a random, auto-similar and repetitive way. For this reason, it is really important to know the hierarchical and relative positions of the elements that compound roman masonry structures.

We have identified two hierarchical key concepts to understand the morphological and topological nature of roman walls: Opus and Structura (Lugli, 1957; Adam, 2006). In general, the first concept was used to indicate any construction methods and in the common language it was preferred to the second one, used in the erudite language. 
Specifically key concepts are:

Opus, identifying the constructive techniques based on composition rules.

Structura, indicating the internal nucleus of a wall and the connection with its wall facing based on the material type.

In our research, we leave out the second concept because at this time our investigation deals with an analysis of the surfaces. We focused on the classification of opus based on the basic interconnections that can lead to a descriptive system used for their unequivocal identification and design.

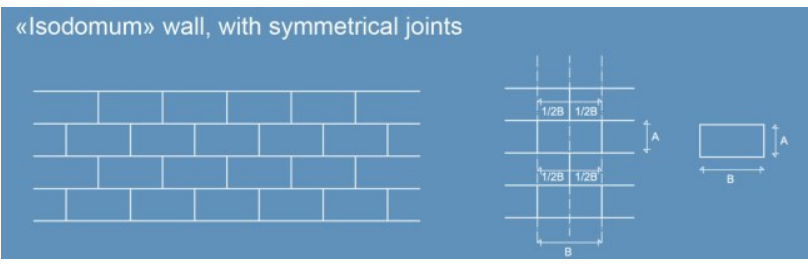

Figure 2. The opus parameters

The parameters adopted to create a cognitive model of opus masonry are: 1) geometric shapes and dimensions of stones or bricks, 2) presence of intervals amongst stones and bricks, and 3) stones and bricks installation (row horizontality and vertical staggering).

\subsection{Creation of the Opus Library}

The individuation of opus typologies on the masonry structures it is made possible by a Maya Plug-in in MEL (Maya Embedded Language) (Cappellini et al., 2012) linked with Nubes web platform (Stefani et al, 2011).

This approach is based on association of the depth map to orthophoto to obtain a $2.5 \mathrm{D}$ model. From this model, it is possible to design the parts of the wall in which are present the different opus typologies and associate a pattern to them that contains the geometric values and composition rules of roman opus. These drawn samples are the entities that the user can interrogate to know the dimensional/parametric values of the elements. They form the library of specific masonry structures.



Figure 3. 3D information system for opus documentation

This phase of our research is supported by an automatic, accurate, flexible and open-source photogrammetric pipeline named Pastis Apero Micmac - PAM, developed by IGN, Paris (Pierrot-Deseilligny et al, 2011). We have employed it to generate ortho-images from non-oriented images, using a user- friendly interface implemented by CNRS Marseille (France). The IGN's pipeline (Pastis Apero Mic Mac - PAM) is subdivided in three main steps to compute: 1) tie points from unordered images using an algorithm named Sift (Pastis); 2) the camera orientation (Apero); and 3) depth maps and point clouds from oriented photos (Mic Mac). A detailed description of this approach has already been presented in (Pierrot-Deseilligny et al., 2011), and several other case studies are available on the TAPEnADe web site (TAPEnADE Project). Moreover, we have specified the information regarding 3D surveying and modelling procedures of some roman walls within the Pompeii archaeological site.

\subsection{A Tool for the Creation of a Parametric Model}

The presented application, currently under construction, has developed with an open source programming language called Processing, useful for visual, animated or static, 2D or 3D, interactive creations. Using this computer language, a Java environment has been developed. Our protocol considers different steps. Firstly, it is possible to import a library of specific masonry structures based on rules belonging to the construction roman methods; secondly, the selected configuration can be dragged-and-dropped onto a black-andwhite raster mask, obtained by an ortho-photo; thirdly, the user can interactively manipulate and adapt the position and the dimensions of the masonry structures and the intervals amongst bricks; finally, we can export these parametric elements in Maya and obtain a $3 \mathrm{D}$ model that contains the geometric values and the composition rules of roman opus.

In a future step, the main improvement will be to drag-and-drop and adapt the parametric elements directly onto ortho-photos.

The essential part of our research is constituted by the library containing the drawn samples that can be queried by users to know the geometric/parametric values of elements. For many types of opus such as opus quadratum or opus vittatum, recognizing the geometric rules is a simple task. However, in other cases such as the one of opus incertum and opus siliceum, establishing the relations amongst elements is more complex: in these last cases, a possibility in future research will be to use a more appropriate system such as Voronoi tessellation and/or Delaunay triangulation.

In fact, firstly the masonry elements are created in parametric and interactive way, and then they are adapted to the photogrammetric data.

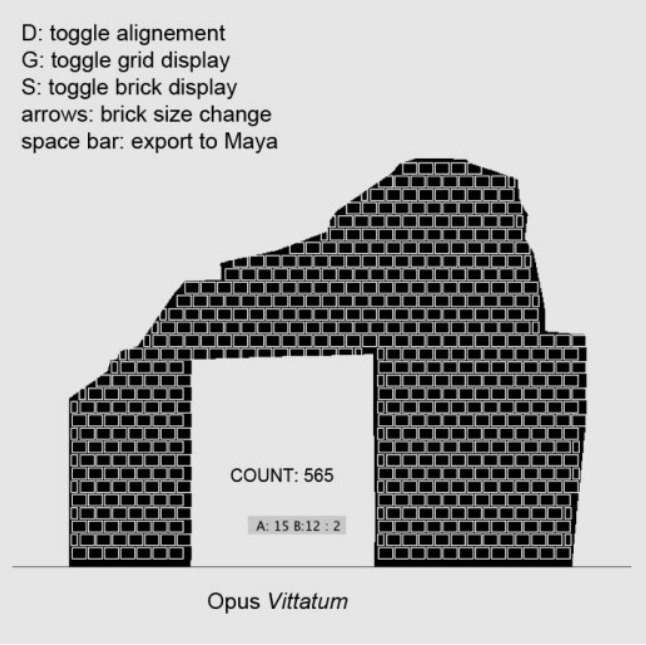

Figure 4. Opus Vittatum represented by Processing 


\section{CONCLUSION AND FUTURE WORK}

Procedural modelling is a useful and time-saving method to simplify and to automate the 3D modelling procedures. His application in archeological field reveals interesting potentialities for the automatic generation and the creation of a library of elements.

In this paper we have presented a method for 3D reconstruction of masonry structures starting from the semantic recognition of the parameters belonging to the construction methods used in roman times. This phase is supported by automatic procedure to get geometrical information and it bases on use of procedural modelling to generate interactively various opus typologies.

Therefore, even if the procedural modelling reveals an accuracy level inferior to the one obtained by manual modelling (brick by brick), this method can be useful when taking into account the static evaluation on buildings (requiring quantitative aspects) and metric measures for restoration purposes.

With this starting point, future research should concentrate on the investigation of image analysis to extract the meaningful information from images such as the boundary lines or points. The aim of this future approach is to match the procedural model with photogrammetric model by the process of images interference.

\section{References}

Adam, J.P., 2006. L'arte di costruire presso i romani, Longanesi, Milano.

Cappellini, V., Nony, N., Stefani, C., De Luca, L., 2012. Surveying Masonry Structures by Semantically Enriched 2.5D Textures: a new approach, M.Ioannides et al. (Eds.): Proceedings of EuroMed 2012, Limassol, Cyprus, LNCS 7616, pp. 729-737, Springer, Heidelberg.

Chevrier, C., Maillard, Y., Perrin, J.P., 2011. A method for the $3 \mathrm{~d}$ modelling of historic monuments: the case of a gothic abbey

http://www.isprs.org/proceedings/XXXVIII/5W1/pdf/chevrier_etal.pdf

Ferdani, D., Pescarin, S., 2011. Dal Gis alla ricostruzione 3D del paesaggio urbano antico.

http://academia.edu/1884152/Dal_Gis_Alla_ricostruzione_3 D_del_paesaggio_urbano_antico

Guidi, G., Remondino, F., Russo, M., Rizzi, A., Voltolini, F., Menna, F., Fassi, F., Ercoli, S., Masci, M.E., Benedetti, B., 2008. A multi-resolution methodology for archeological survey: the Pompeii Forum. Proc. of 14th Int. Conference on Virtual Systems and MultiMedia (VSMM), pp. 51-59, Limassol, Cyprus

Havemann, S., Generative Mesh Modeling. Dissertation, TU Braunschweig, 2005

http://www.generativemodeling.org/GenerativeModeling/DO CUMENTS

Haegler S., Muller P., Van Gool L., 2009. Procedural Modeling for Digital Cultural Heritage. EURASIP Journal on Image and Video Processing, Vol. 2009.
Lugli, G., 1957. La tecnica edilizia romana, Bardi, Roma.

Muller P., Wonca P., Ulmer A., Van Gool L., 2006. Procedural Modeling of Buildings. ACM Siggraph, New York, pp. 614-623

Parish Y., Muller, P., 2001. Procedural Modeling of the Cities, ACM Siggraph, NewYork , pp. 301-308.

Pescarin, S., 2009. Reconstructing Ancient Landscapes, Budapest.

Pierrot-Deseilligny, M., De Luca, L., Remondino, F., 2011. Automated image-based procedures for accurate artifacts 3D modeling and orthoimage generation. In 23th Int. CIPA Symposium, Prague, Czech Republic (2011)

Pierrot-Deseilligny, M., Cléry, I., 2011. APERO, an Open Source Bundle Adjusment Software for Automatic Calibration and Orientation of a Set of Images. In Proceedings of the ISPRS Commission V Symposium, Image Engineering and Vision Metrology, Trento, Italy (2011)

Stefani C., Brunetaud X., Badosa S., Beck K., De Luca L., Al-Muktar M., 2011. Developing a toolkit for mapping and display stone alteration on a web-based documentation platform, Journal of Cultural Heritage, Elsevier.

TAPEnADe project (Tools and Acquisition Protocols for Enhancing the Artifact Documentation) : http://www.tapenade.gamsau.archi.fr 


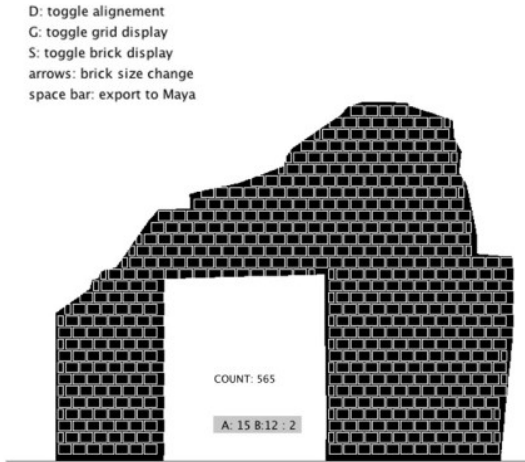

Opus Vittatum

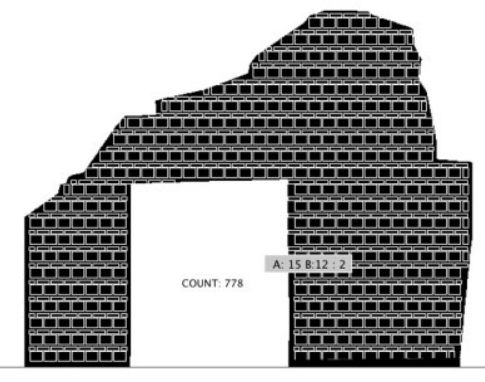

Opus Vittatum mixtum

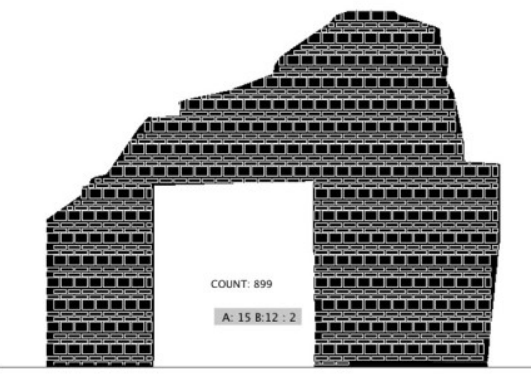

Opus Vittatum mixtum

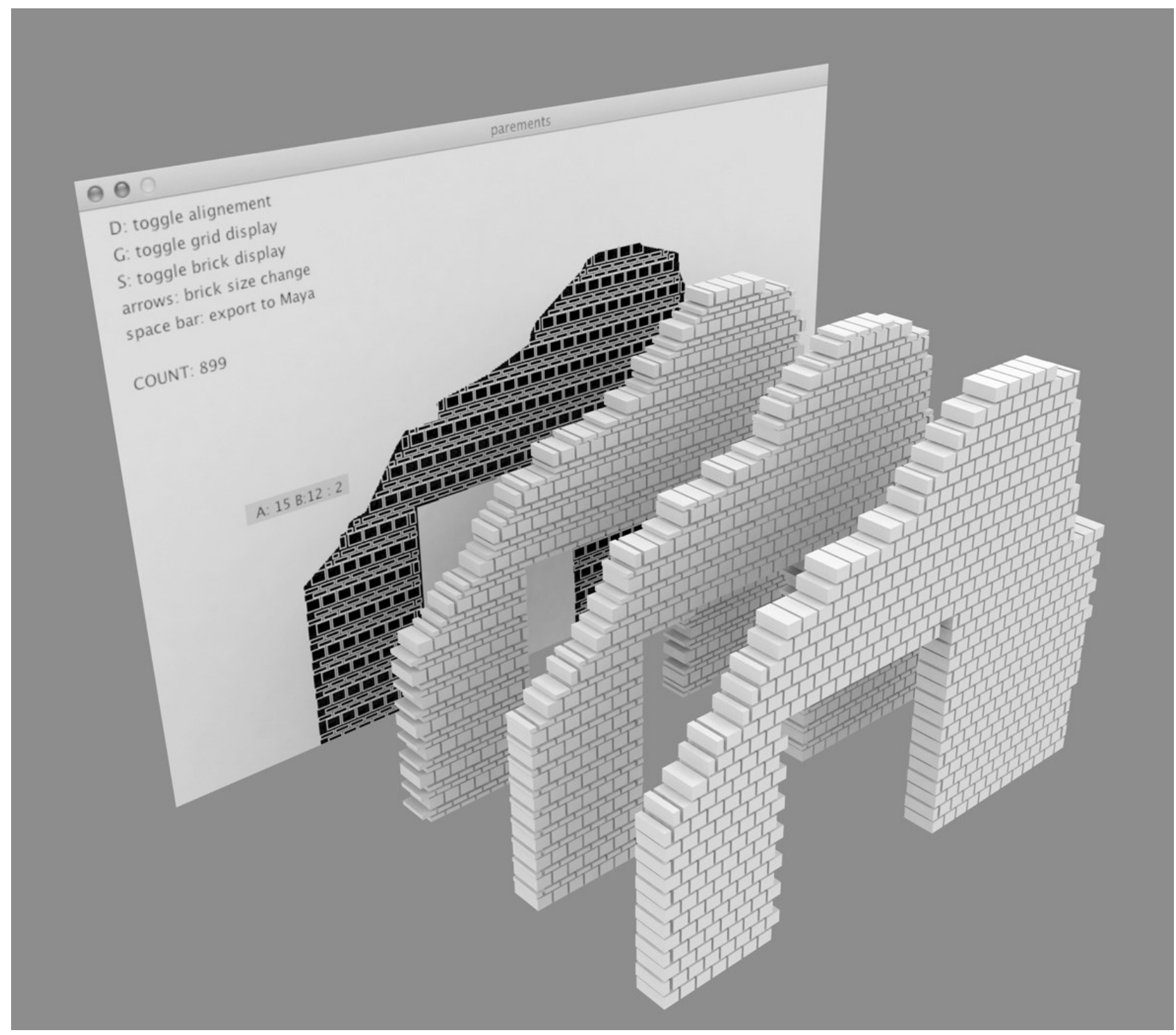

Figure 5. Opus types: from Processing to 3D Maya representation 